\title{
Zwei Drittel der Kassen haben Beiträge zum Jahreswechsel erhöht
}

Das Gesundheitsreformjahr 2015 liegt zurück, noch gibt es keine endgültigen Zahlen zu Gesamtausgaben der Gesetzlichen Krankenversicherung (GKV), doch das Bundesgesundheitsministerium (BMG) zieht schon mal eine recht positive Bilanz: „Die Entwicklung des ersten bis dritten Quartals 2015 bietet auf Basis der bei den Krankenkassen und dem Gesundheitsfonds vorhandenen Finanzreserven eine solide Ausgangsbasis für die Finanzentwicklung der GKV in 2015 und in den Folgejahren“, heißt es in einer Erklärung des BMG. Die ersten neun Monate 2015 hat die GKV insgesamt mit einem Defizit abgeschlossen, verfügt allerdings immer noch über hohe Reserven, wie die Zahlen des BMG zeigen.

Die Ausgaben der GKV sind demnach in fast allen Bereichen gestiegen. Für die zahnärztliche Behandlung und Zahnersatz betrug der Anstieg in den ersten drei Quartalen des vergangenen Jahres 2,9 und 1,5 Prozent. Da die Angaben jedoch in den Leistungsbereichen nicht vollständig vorlagen, haben die Veränderungsraten laut BMG „,noch erheblichen Schätzcharakter“. Auf Basis der Mitte Oktober 2015 vom GKV-Schätzerkreis erfolgten Prognosen zur Einnahmen- und Ausgabenentwicklung der GKV hatte sich für das Jahr 2016 eine Erhöhung der Krankenkassenbeiträge um weitere 0,2 Prozentpunkte ergeben, um die laufenden Kosten auch künftig decken zu können. Zum Jahreswechsel konnte nun jede Krankenkasse selbst entscheiden, ob sie einen Zusatzbeitrag von ihren Mitgliedern erhebt und wie hoch dieser ausfällt. Nach Angaben der Stiftung Warentest haben zum Jahreswechsel 59 der frei wählbaren Kassen ihre Beiträge erhöht - etwa ein Drittel der Krankenkassen haben auf eine Beitragserhöhung verzichtet. Bei den meisten Kassen liegt die Steigerung, die finanziell von den Arbeitnehmern geschultert werden muss, zwischen 0,2 und 0,4 Prozentpunkte. Allerdings gibt es einige „Ausreißer“, die ihre Beiträge um 0,6 oder sogar 0,8 Prozentpunkte erhöht haben. Patienten haben die Möglichkeit, innerhalb von zwei Monaten nach der Erhöhung von ihrem außerordentlichen Kündigungsrecht Gebrauch zu machen und die Kasse zu wechseln. Der gesetzlich festgelegte Krankenkassenbeitrag liegt seit 2015 bei 14,6 Prozent des monatlichen Bruttoeinkommens plus einem möglichen Zusatzbeitrag. Vom gesetzlich festgelegten prozentualen Anteil zahlen Arbeitgeber und Arbeitnehmer je die Hälfte. Während der Arbeitgeberanteil bei 7,3 Prozent eingefroren ist, zahlen die Krankenversicherten den Mehrbedarf ihrer Kasse durch den Zusatzbeitrag allein. Für dieses Jahr ergibt sich ein durchschnittlicher Zusatzbeitrag von 1,1 Prozent.

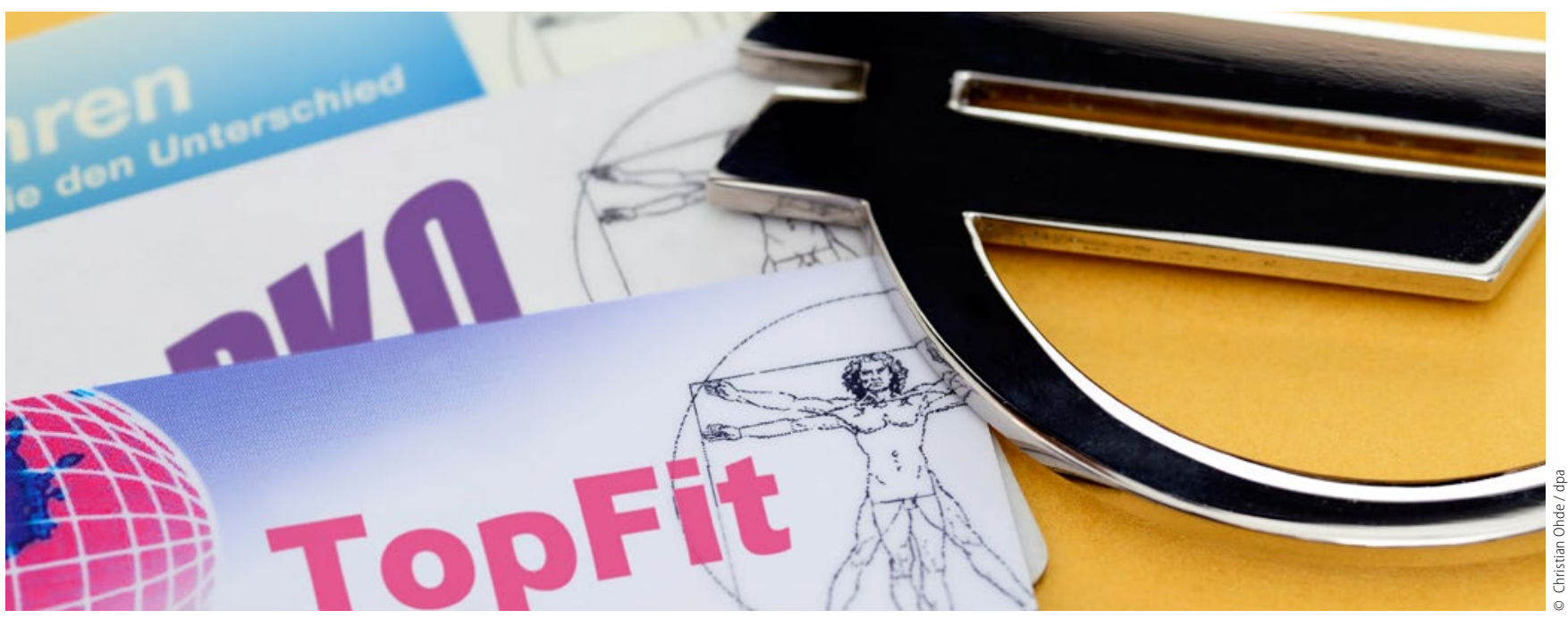

\section{Private Krankenversicherung}

\section{Zahnzusatzversicherungen liegen bei Patienten weiter im Trend}

Immer mehr Menschen in Deutschland bauen auf die private Krankheitsvorsorge: Im Bereich der Zusatzversicherungen zur Gesetzlichen Krankenversicherungen legten die privaten Krankenversicherungen (PKV) auch im vergangenen Jahr (Berichtszeitraum 2014) erneut zu. Dies geht aus dem jüngsten Zahlenbericht des PKV-Verbandes hervor. Rund 24,3 Millionen Zusatzverträge haben die Deutschen abgeschlossen. Spitzenreiter mit 14,4 Millionen Verträgen sind die Zahnzusatzversicherungen. Fast vier Milliarden Euro leisteten die Versicherer 2014 aus den Zahn-Tarifen. 2,3 Milliarden Euro wurden für Zahnersatz gezahlt, Zahnbehandlungen schlugen für die privaten Versicherer mit 1,4 Milliarden Euro zu Buche. Auf kieferorthopädische Leistungen entfielen 256 Millionen Euro. Die Zahl der abgeschlossenen Zahn-Policen hat sich innerhalb von zehn Jahren etwa verdoppelt. Die ambulanten Tarife und die Tarife für Wahlleistungen im Krankenhaus folgen weit dahinter. Die Zahl der privat Vollversicherten ist dagegen seit einigen Jahren rückläufig. 